\title{
Initiation of Pharyngeal Swallow with Bolus Head in Pyriforms
}

National Cancer Institute

\section{Source}

National Cancer Institute. Initiation of Pharyngeal Swallow with Bolus Head in Pyriforms.

NCI Thesaurus. Code C127223.

A finding of pharyngeal swallow initiated with bolus head in pyriforms. 